\title{
Gait Analysis Using a Shoe-Integrated Wireless Sensor System
}

\author{
Stacy J. Morris Bamberg, Member, IEEE, Ari Y. Benbasat, Member, IEEE, Donna Moxley Scarborough, \\ David E. Krebs, and Joseph A. Paradiso, Senior Member, IEEE
}

\begin{abstract}
We describe a wireless wearable system that was developed to provide quantitative gait analysis outside the confines of the traditional motion laboratory. The sensor suite includes three orthogonal accelerometers, three orthogonal gyroscopes, four force sensors, two bidirectional bend sensors, two dynamic pressure sensors, as well as electric field height sensors. The "GaitShoe" was built to be worn in any shoe, without interfering with gait and was designed to collect data unobtrusively, in any environment, and over long periods. The calibrated sensor outputs were analyzed and validated with results obtained simultaneously from the Massachusetts General Hospital, Biomotion Laboratory. The GaitShoe proved highly capable of detecting heel-strike and toe-off, as well as estimating foot orientation and position, inter alia.
\end{abstract}

Index Terms-Biomedical measurements, body sensor networks, legged locomotion, multisensor systems, telemetry.

\section{INTRODUCTION}

C LINICAL gait analysis is the investigation of the pattern of walking. At present, gait analysis is primarily carried out in one of two ways: in a motion laboratory, with full analysis of the motion of body segments using highly accurate computerbased force and optical tracking sensors, or in an office with the clinician making visual observations. The first method is expensive, requires the maintenance of a dedicated motion laboratory, and uses cumbersome equipment attached to the patient, but produces well-quantified and accurate results for short-distance ambulation. The second method is inexpensive and does not require special equipment, but requires additional time from the clinician, and the results are qualitative, unreliable, and difficult to compare across multiple visits.

There is a need for an alternative analysis method that is capable of providing quantitative and repeatable results over extended time periods. A system that can quantitatively analyze

Manuscript received May 31, 2004; revised March 9, 2007. This work was supported in part by the Center for the Integration of Medicine and Innovative Technology, in part by the Whitaker Foundation, and in part by the Things That Think Consortium at the Massachusetts Institute of Technology (MIT) Media Laboratory.

S. J. M. Bamberg was with Harvard/Massachusetts Institute of Technology (MIT) Division of Health Sciences and Technology, Cambridge, MA 02139 USA. She is now with Department of Mechanical Engineering, University of Utah, Salt Lake City, UT 84112 USA (e-mail: sjm@alum.mit.edu).

A. Y. Benbasat and J. A. Paradiso are with the Massachusetts Institute of Technology (MIT) Media Laboratory, Cambridge, MA 02139 USA (e-mail: ayb@alum.mit.edu; joep@media.mit.edu).

D. M. Scarborough is with the Massachusetts General Hospital (MGH) Orthopedic Biomechanics and Biomaterials Laboratory, Boston, MA 02114 USA (e-mail: dscarborough@partners.org).

D. E. Krebs, retired, was with the Massachusetts General Hospital (MGH) Institute of Health Professions, Boston, MA 02129 USA (e-mail: dkrebs@mit.edu).

Digital Object Identifier 10.1109/TITB.2007.899493 gait for patients offers clinicians and patients new opportunities for diagnosis and treatment of chronic walking problems. There has been considerable previous work in both research and commercial spheres focused on the development of more mobile methods of analyzing gait. The advantage of directly measuring the pressure distribution beneath the foot drove many early shoe-based systems. The shrinking size of data storage has further encouraged the development of untethered systems.

In 1990, Wertsch et al. [1] developed a tethered system for measuring the pressure distribution beneath the foot, using seven force-sensitive resistors (FSRs), and used this device to quantify the differences between shuffling and walking [2], and identified the need to collect data over a long time period in populations with large step-to-step variations in gait [3]. In 1994, Hausdorff et al. [4], [5] developed a system capable of detecting several of the temporal gait parameters with two FSRs positioned under the heel and in the general area under the toes and metatarsals, connected to a circuit board and battery pack worn on the ankle, which has been used to evaluate the likelihood of falling in the elderly [6] and to find patterns in gait [7].

More recent research has been driven by subspecialty interests in gait analysis. For diabetics, Morley et al. [8] and Maluf et al. [9] developed an insole-based system to quantify the conditions inside the shoe, to predict the progression of skin breakdown and ulceration in diabetic patients with peripheral neuropathy. Pappas et al. [10], [11] used a pattern recognition algorithm to define the transitions during the gait cycle using their device consisting of three FSRs located on an insole (one under the heel, and two at the first and fourth metatarsal heads), and a gyroscope. The system was tested on two subjects with incomplete spinal injury and was used to trigger functional electrical stimulation (FES), with demonstrated benefit for both subjects.

Vildjiounaite et al. [12] developed a real-time system, using accelerometers and magnetic sensors. The magnetic sensor data were used to determine foot orientation and identify steps; the averaged peak forward acceleration was used with a lookup table to estimate the step length. For level walking, location was estimated with an error of $5 \%$.

Other research devices include instrumented walkways [13], "piezodyanomometric" platforms [14], or instrumented floors [15], [16]. Such systems can only provide information while the subject walks on the platform. In addition, research platforms have been developed to recognize gait without instrumenting the subject, primarily by videotape analysis [17]-[21], and also through the use of radar [21], [22]. 
TABLE I

SENSORS SELECTED FOR THE GAITSHOE

\begin{tabular}{|c|c|c|c|c|}
\hline Sensor Type & Parameters & Sensor Output & Range & Part Number(s) [Manufacturer] \\
\hline Accelerometers & $\begin{array}{l}\text { Stride length and stride velocity, and } \\
\text { other velocities and displacements }\end{array}$ & $\begin{array}{l}\text { Voltage change corresponding to acceleration; } \\
\text { single integration of acceleration yields velocity, } \\
\text { double integration yields distance (integration } \\
\text { done after correcting for gravitational component). }\end{array}$ & $\pm 2 \mathrm{~g}$ & ADXL202E [Analog Devices] \\
\hline Gyroscopes & Orientation & $\begin{array}{l}\text { Voltage change corresponding to angular velocity; } \\
\text { single integration yields angle of rotation. }\end{array}$ & $\begin{array}{l} \pm 150 \% \mathrm{sec} \\
\pm 300 \% \mathrm{sec}\end{array}$ & $\begin{array}{l}\text { ADXRS150 [Analog Devices] } \\
\text { ENC-03J [Murata] }\end{array}$ \\
\hline Force sensitive resistors & $\begin{array}{l}\text { Force distribuition under foot, and } \\
\text { heel-strike timing and toe-off timing }\end{array}$ & $\begin{array}{l}\text { Resistance change corresponding to applied force } \\
\text { across the sensor, resulting from change in } \\
\text { compression of the sensor. }\end{array}$ & $\begin{array}{l}1 \text { to } 100 \mathrm{~N} \\
1 \text { to } 100 \mathrm{~N}\end{array}$ & $\begin{array}{l}\text { FSR-400 [Interlink Electronics] } \\
\text { FSR-402 [Interlink Electronics] }\end{array}$ \\
\hline Polyvinylidene fluoride strips & Heel-strike timing and toe-off timing & $\begin{array}{l}\text { Voltage change corresponding to dynamic pressure } \\
\text { across the sensor. }\end{array}$ & & LDT0 [Measurement Specialties] \\
\hline Bend sensors & $\begin{array}{l}\text { Plantar flexion/ dorsi- flexion } \\
\text { Flexion at metatarsals }\end{array}$ & $\begin{array}{l}\text { Resistance change corresponding to flexion angle, } \\
\text { resulting from strain of the sensor. }\end{array}$ & $0^{\circ}$ to $90^{\circ}$ & FLX-01 [The Images Co.] \\
\hline Electric Field Sensor & Height of foot above floor & Capacitance corresponding to distance. & 10 to $100 \mathrm{pF}$ & MC33794DH [Motorola] \\
\hline
\end{tabular}

Commercial systems are numerous, and cover a wide range of applications, from tap dance (Yamaha's Miburi system) [23] to golf-swing analysis [24]. A very popular application is the use of inertial sensors to provide athletes with information, such as the products available from Reebok [25], FitSense [26], Vectrasense [27], Adidas [28], and Nike [29]. National Center for Supercomputing Applications (NCSAs) Cyberboots provide walking interaction in a virtual reality environment [30]. For medical applications, Tekscan and Clevemed, among others, have developed insoles that measure pressure distribution [31], [32]. The "IDEEA LifeGait System" from Mini-Sun uses wired accelerometers distributed on the body to determine parameters of gait and motion [33].

The research presented in this paper developed from the Expressive Footware project by Dr. Paradiso and students in the Responsive Environments Group at the Massachusetts Institute of Technology (MIT) Media Laboratory [34]. That project resulted in a pair of running shoes that were each equipped with a wireless sensor board and an instrumented insole that were designed for control, rather than measurement. Dancers wore the shoes to directly manipulate real-time interactive musical outputs, generated by a computer that interpreted the base-station data stream with an elaborate rule base. It reached high acclaim in the dance community, and was recognized with the Discover Award for Technical Innovation in 2000 [35].

Our research sought to create a system to provide instrumented wireless gait analysis outside of traditional, expensive motion laboratories. We named the system "GaitShoe." Such a system has the potential to be highly informative by allowing data collection throughout the day in a variety of environments, thus providing a vast quantity of long-term data not obtainable with current gait analysis systems. The "GaitShoe" system has been designed with components configured to minimally affect gait, and is readily fixed on typical athletic shoes.

The structure of this paper is as follows. Section II describes the hardware and sensors used in the GaitShoe. Patient testing, selection, and recruitment are discussed in Section III. Results are presented in Section V and discussed in Section VI.

\section{HARDWARE/SENSORS}

\section{A. Sensor Selection}

The sensors used in the GaitShoe were selected with the goal of creating a highly instrumented system capable of sensing many parameters that characterize gait, and are summarized in Table I; details of the implementations are contained in [36]. A schematic of the GaitShoe is shown in Fig. 1, with labels indicating relevant anatomical markers.

For the analysis of the kinematic motion of the foot, two dualaxis accelerometers and three gyroscopes were placed at the back of the shoe, oriented such that the individual sensing axes were aligned along three perpendicular axes. Such a device is known as an inertial measurement unit (IMU); this realization is termed a strapdown IMU [37], as its measurement coordinates are fixed to the frame of the shoe. This application used two dual-axis accelerometers, the microelectromechanical system (MEMS)-based Analog Devices ADXL202E, and two types of gyroscopes: the MEMS-based Analog Devices ADXRS150, and the vibrating-reed-based Murata ENC-03 J (these devices sense in orthogonal planes, which facilitated the construction of a flat IMU package).

To assess the timing parameters and pressure distribution, FSRs and polyvinylidene fluoride (PVDF) strips were placed under the foot. Two sizes of FSRs manufactured by Interlink Electronics were used: two FSR-402 (diameter, $5 \mathrm{~mm}$ ) placed underneath the heel pad, one medially and the other laterally, and two FSR-400 (diameter, $12.7 \mathrm{~mm}$ ) were placed under the first and fifth metatarsal heads (each subject's foot was palpated and inked, and the ink was transferred to paper; these markings were used to set the location of the FSRs and the PVDF strips in the insole). The PVDF strips are piezoelectric sensors, and were configured to provide an output corresponding to dynamic pressure. Two PVDF strips, part LDT0 from Measurement Specialties, were placed under the heel and the great toe.

A bidirectional bend sensor was used to analyze flexion during gait. Two FLX-01 sensors (unidirectional resistive bend sensors manufactured by The Images Company) were placed 


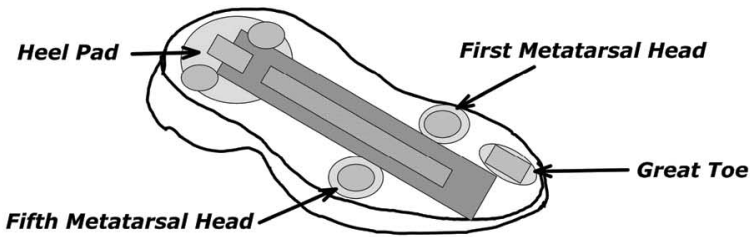

\section{Insole contains:}

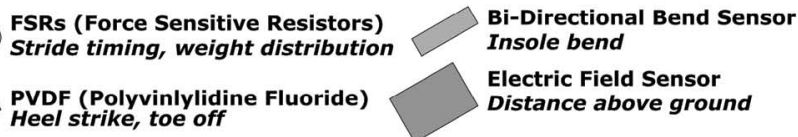

Fig. 1. Schematic of the GaitShoe system.

back to back, and a differential amplifier was implemented to combine the individual outputs into a bidirectional bend output. One was located at the back of the heel and held next to the shin by an anklet, to evaluate plantar flexion and dorsiflexion. The other was located in the insole, and measured flexion at the metatarsal-phalangeal joint.

A capacitive sensor was developed later, using a multielectrode electric-field imaging device (MC33794DH) manufactured by Motorola, and was added to investigate the utility of using a more direct method of measuring the elevation of the foot via capacitive loading from the floor [38]. As noted in Fig. 1, the range and angle between the feet were measured by timing the transit of an ultrasound ping from one shoe to the other. Preliminary results showed promise [36], but this system was not sufficiently mature to use during validation.

\section{B. Physical Implementation}

The mechanical hardware was designed to accommodate the sensors located beneath the foot, all of the electronics (including the sensors located at the back of the shoe), an antenna for the wireless transmission, and the power supply.

The GaitShoe system comprised two shoe modules and a base station. Each shoe module consisted of an instrumented insole placed beneath the foot and an attachment that mounted to the back of the shoe. A sample insole is shown in Fig. 2, and enclosed sensors are detailed in Fig. 1.

The shoe attachments contained the IMU sensors, microcontroller (Silicon Laboratories C8051F206), wireless transceiver (RF Monolithics DR3000-1), antenna, power supply, and conditioning electronics, and were implemented on a stack of printed circuit boards [39], [40]. The shoe attachments were designed with the bulk of the volume located behind the heel, to minimize the effect on foot motion. The attachment was made from 0.125 in sheets of polyethylene terephthalate glycol (PTG), a thermoformable, machinable, and shatter-resistant material. A photo of the attachments and insoles is shown in Fig. 3.
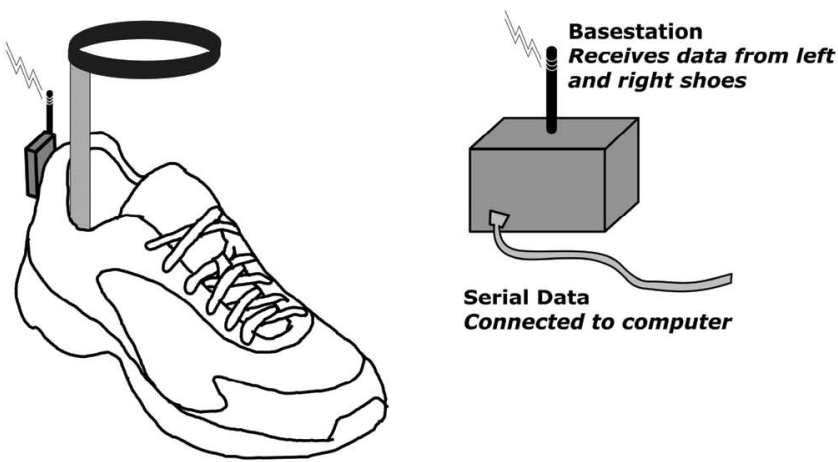

Shoe attachments contain:

Circuit Boards, Power Supply, RF Transceiver/Antenna, and:

- 3 Axes of Gyroscopes Angular velocity

- 3 Axes of Accelerometers - Ultrasound Sensor, Board to Board Linear acceleration

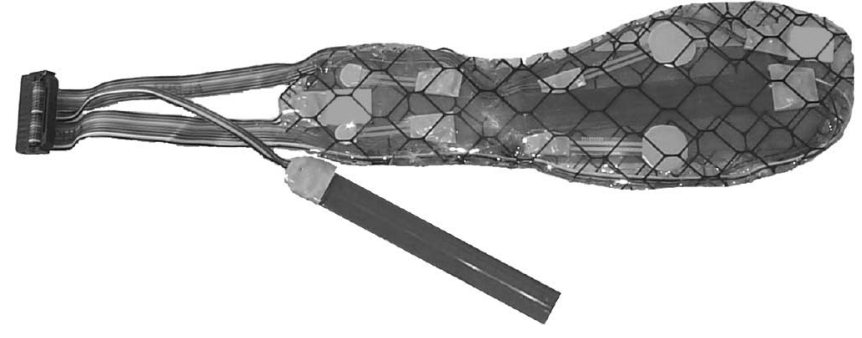

Fig. 2. Sample GaitShoe insole.

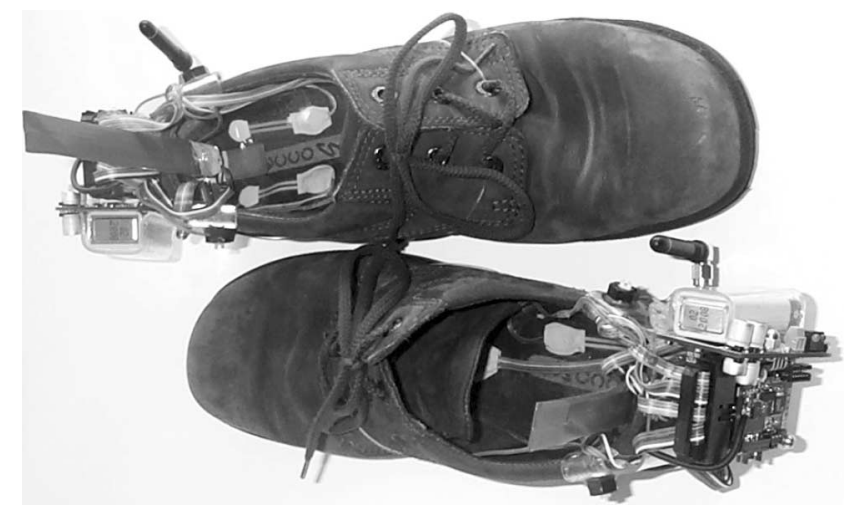

Fig. 3. GaitShoe hardware mounted on shoes.

The base station consisted of a metal box with an antenna mounted externally, housing the circuit board with the microcontroller and the power board, plus an additional board with a MAX233 serial line level converter chip to drive a conventional RS-232 cable. An optoisolated trigger from the Massachusetts General Hospital (MGH) Biomotion Laboratory's (BML) equipment was input via a BNC connector and routed to the microcontroller, providing a commonly observed reference that was used to align the time scales of the two individual systems. The microcontroller in the base station looped through a 
simple time-division multiplexing routine to poll each shoe and receive its data.

1) RF Transmit: Send hex byte 6C (poll left shoe).

2) $R F$ Receive: Listen for $6 \mathrm{~ms}$.

3) RF Transmit: Send hex byte E8 (poll right shoe).

4) $R F$ Receive: Listen for $6 \mathrm{~ms}$.

The left shoe's microcontroller looped through the following routine.

1) Sample data from all sensors.

2) RF Receive: Listen until hex byte $6 \mathrm{C}$ received.

3) $R F$ Transmit: Send data.

The right shoe's microcontroller followed the same overall routine as the left, but listened for hex byte E8 in step 2 (these header bytes were chosen arbitrarily, and had an equal number of high and low bits). Thus, each shoe collected analog data from the sensors while the other was sending, then waited to receive its own code before transmitting to the base station.

The receive line on the base station was connected directly to the serial converter, immediately relaying the data to the computer. Each shoe sent a full update every $13.4 \mathrm{~ms}$, corresponding to a net data transmission rate (for each shoe) of approximately $75 \mathrm{~Hz}$ per shoe. All sensor signals were normalized to a scale of $0-1$ by dividing them by the maximum 12-bit analog-to-digital converter value of 4095 .

\section{SubJeCt Testing OvervieW}

\section{A. Testing Systems}

Volunteers were recruited for the validation of the GaitShoe system. The testing involved placing the GaitShoe instrumentation on the subjects' own walking shoes. Every subject underwent simultaneous gait evaluation using the MGH BMLs Selspot II (Selective Electronics, Partille, Sweden) data acquisition system.

The Selspot II serially sampled infrared LEDs, arranged in wired arrays, at a rate of $152 \mathrm{~Hz}$. The LED arrays were placed on 11 body segments (bilaterally: feet, shanks, thighs, arms; and the pelvis, trunk, and head). The TRACK kinematic data analysis software package was used reconstruct the 6 DOF kinematics of the arrays [41]. Within the $2 \mathrm{~m}^{3}$ viewing volume, this system is capable of defining the 3-D positions of each body segment within $1 \mathrm{~mm}$, and the three orientations within $1^{\circ}$. Two piezoelectric force plates (Kistler Instruments Type $9281 \mathrm{~A}$, Winterthur, Switzerland) were used to acquire ground reaction forces. As set in the MGH BML, this system has an accuracy of $\pm 1 \%$ of full scale, which corresponds to $\pm 10 \mathrm{~N}$ of vertical force for forces and frequencies encountered during gait [42], [43].

\section{B. Subject Recruitment and Consent}

Each subject was asked to perform a series of locomotor tasks, while both systems simultaneously collected data. The parameters collected from the two systems were analyzed and compared to validate the GaitShoe. The subjects with healthy gait were recruited by word of mouth and comprised a mixture of colleagues and students from the MIT and MGH commu-
TABLE II

SUMMARY OF SUBJECT CHARACTERISTICS

\begin{tabular}{lll}
\hline \hline & Healthy gait & Parkinsonian gait \\
\hline Genders & 3 males, 7 females & 2 males, 3 females \\
Age [years] & $32.1(24.9-54.0)$ & $65.3(53.8-76.4)$ \\
Height $[\mathrm{m}]$ & $1.7(1.6-1.8)$ & $1.7(1.6-1.8)$ \\
Weight $[\mathrm{kg}]$ & $66.7(48.2-115.0)$ & $71.3(52.3-94.5)$ \\
\hline \hline
\end{tabular}

nities. Collaborating physicians from the MGH Department of Neurology recruited the subjects with Parkinson's disease (PD).

Subjects with PD were included for the purpose of acquiring data in a population with known gait dysfunction. As indicated in recent research, persons often perform gait at their "best" when being tested in a formal laboratory setting, yet they and their family members report poor gait ability throughout their day when at home. This is particularly common among persons with PD. Persons with PD exhibit changes in limb stiffness that influences gait ability throughout the day, not only as a result of the underlying disease but also from the medications that control the symptoms of Parkinsonian movement disorders. Therefore, this patient population should benefit from home gait evaluation, which can provide information about gait abnormalities present in everyday life that have not traditionally been captured in analyses carried out in motion laboratories [44].

All subjects were adults who could understand and follow basic directions. Persons were excluded if they reported acute pain that prevented the performance of their comfortable, typical movement, or if an unstable medical condition such as uncontrolled hypertension or diabetes mellitus was present. Both the MGH Institutional Review Board (IRB) and the MIT Committee on the Use of Humans as Experimental Subjects (COUHES) approved the protocols for this study. All the subjects provided written consent in accordance with the MGH IRB and the MIT COUHES.

\section{Overview of Participants}

A total of 16 subjects were recruited for the validation of the GaitShoe. Characteristics for each group are summarized with means (and standard deviations) in Table II.

\section{Testing Protocol}

Each subject first walked at his or her own self-selected natural pace for 2 to 4 trials, termed "free gait." Next, a number of calibration routines were carried out, including a chair rise trial, wherein the subject stood from a seated position. Following the calibrations, another 2 to 4 trials of "free gait" were collected followed by ten other trials of various walking activities [36].

\section{SENSOR CALIBRATION AND ANALYSIS}

The full output from the GaitShoe system is shown in Fig. 4. As the sensor specifications given by the manufacturers were generally not sufficiently accurate for our applications, all components were individually calibrated [36]. The calibration and the $z$-gyroscope was integrated to determine the pitch of the 


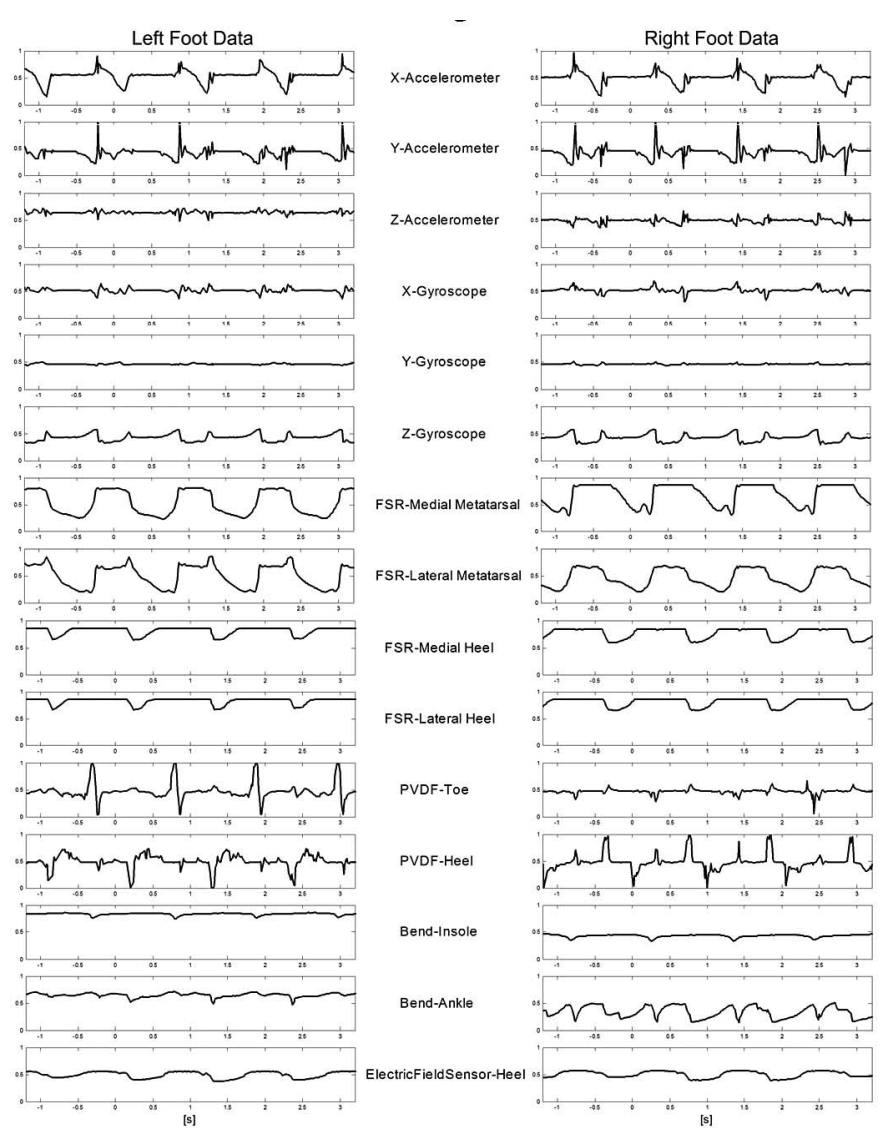

Fig. 4. Uncalibrated outputs of all GaitShoe sensors.

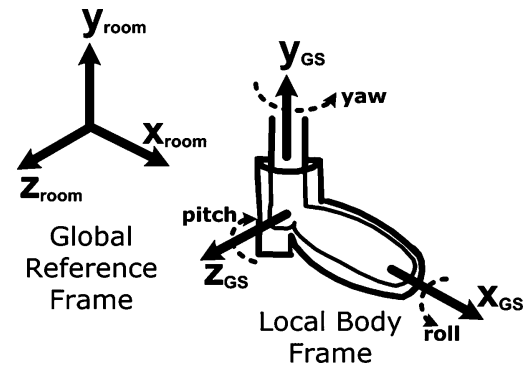

Fig. 5. Reference frames.

foot, the $x$ - and $y$-accelerometers were combined and integrated to determine stride length, and information from the gyroscopes and accelerometers were used to finalize the heel-strike and toe-off timing results.

\section{A. Analysis Model}

The relevant coordinate systems used for the analysis of the data are shown in Fig. 5. The first corresponds to the global reference frame of the room and the second corresponds to the local body frame, in which the sensors are mounted and collect their measurements. The determination of room-based parameters, such as orientation or position, requires a transformation to the global reference frame.

The initial analysis presented here was simplified by assuming that the data collected involved linear motion in the
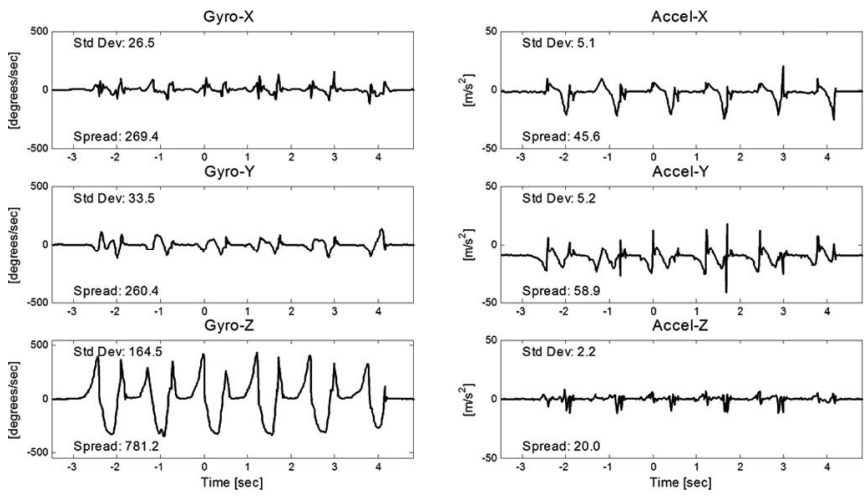

Fig. 6. Comparison of IMU outputs during walking gait.

$X_{\mathrm{GS}}-Y_{\mathrm{GS}}$ plane only (no translation in $Z_{\mathrm{GS}}$ ), and rotation about the $Z_{\mathrm{GS}}$-axis only (the $X_{\mathrm{GS}}-Y_{\mathrm{GS}}$ plane remained parallel to the $X_{\text {room }}-Y_{\text {room }}$ plane). In other words, it was assumed that the motion involved changes only in a single plane. The subject testing involved collecting data during walking, with the subject walking in a straight line only.

As evident in Fig. 6, typically, the angular velocities about $X_{\mathrm{GS}}$ and $Y_{\mathrm{GS}}$ were less than a third of the angular velocity about $Z_{\mathrm{GS}}$, and the acceleration in $Z_{\mathrm{GS}}$ less than half of the accelerations in $X_{\mathrm{GS}}$ and $Y_{\mathrm{GS}}$. Thus, these were reasonable assumptions for an initial analysis of the GaitShoe system, without requiring the complex mathematics necessary to represent true 3-D motion.

\section{B. Calibration}

1) Force-Sensitive Resistors: The FSRs were calibrated using a TA-XT Texture Analyzer from Stable Micro Systems, a curve was fit to the calibration data (a third-order polynomial in an exponential was empirically found to provide a good fit to the data). The relationship between the scaled FSR-402 output, $V_{F 402}$, and the applied force in Newton, $F$, is described by

$$
F=9.8 \exp \left\{-8.7 V_{F 402}^{3}+14.2 V_{F 402}^{2}-10.6 V_{F 402}+2.4\right\}
$$

and the relationship between the FSR-400 output, $V_{F 400}$, and the applied force in Newton, $F$, is described by

$$
F=9.8 \exp \left\{-10.5 V_{F 400}^{3}+21.9 V_{F 400}^{2}-21.6 V_{F 400}+6.8\right\} \text {. }
$$

The $95 \%$ confidence interval of the curve-fit for the FSR-402 was $14.95 \mathrm{~N}$ and for the FSR-400 was $14.64 \mathrm{~N}$. This error is rather large, and is likely due at least in part to the nonlinearity of the FSR response. The output of the FSRs is nonlinear, somewhat due the choice of conditioning electronics. Also, over time and with use, the adhesive layer in the FSRs may break down and contribute to an increased nonlinearity.

2) Gyroscopes: The zero offset of the gyroscopes was the output when the hardware was at rest. The sensitivity of the gyroscopes was determined by rotating each gyroscope about its sensitive axis, through a range of constant angular velocities. The results for the gyroscopes on the two IMU boards used in testing are summarized in Table III. The relative orientations 
TABLE III

GyrosCoPe SENSITIVITIES AND ZERO OFFSETS

\begin{tabular}{lccc}
\hline \hline Gyroscope & \multicolumn{2}{c}{ Sensitivity } & Zero Offset \\
& Slope & Coeff. of Corr. & \\
\hline IMU-1 X & $9.25 \times 10-4$ & 1 & 0.492 \\
IMU-1 Y & $3.21 \times 10-4$ & 0.999 & 0.462 \\
IMU-1 Z & $3.30 \times 10-4$ & 1 & 0.423 \\
IMU-2 X & $9.33 \times 10-4$ & 1 & 0.508 \\
IMU-2 Y & $3.32 \times 10-4$ & 1 & 0.446 \\
IMU-2 Z & $3.23 \times 10-4$ & 1 & 0.432 \\
\hline \hline
\end{tabular}

TABLE IV

ACCELEROMETER SENSITIVITIES AND ZERO OFFSETS

\begin{tabular}{lcccccc}
\hline \hline \multirow{2}{*}{ Accelerometer } & \multicolumn{2}{c}{ +1g Output } & \multicolumn{2}{c}{-1g Output } & Sensitivity & Zero Offset \\
& Mean & Std Dev & Mean & Std Dev & & \\
\hline IMU-1 X & 0.676 & 0.001 & 0.425 & 0.001 & 0.13 & 0.551 \\
IMU-1 Y & 0.694 & 0.002 & 0.442 & 0.002 & 0.13 & 0.568 \\
IMU-1 Z & 0.619 & 0.005 & 0.375 & 0.002 & 0.12 & 0.497 \\
IMU-2 X & 0.716 & 0.002 & 0.467 & 0.002 & 0.13 & 0.591 \\
IMU-2 Y & 0.684 & 0.002 & 0.433 & 0.004 & 0.13 & 0.558 \\
IMU-2 Z & 0.750 & 0.003 & 0.507 & 0.003 & 0.12 & 0.628 \\
\hline \hline
\end{tabular}

between the gyroscopes were not determined beyond their mounting tolerances on the circuit board.

3) Accelerometers: The sensitivity of the accelerometers was determined by rotating the sensors through gravity. The resulting data were low-pass filtered, with second-order Butterworth coefficients and a low-pass cutoff frequency of $2 \mathrm{~Hz}$ (sampling frequency, $75 \mathrm{~Hz}$ ), to remove any small acceleration changes resulting from hand jitter. The rotation by hand was performed slowly (to minimize centripetal acceleration), and was carried out at seven different times throughout the subject testing. The sensitivity was set to the slope of the line between the mean of all $+1 g$ outputs and the mean of all $-1 g$ outputs, and the zero offset was set as the midpoint between the means of the $+1 \boldsymbol{g}$ and the $-1 \boldsymbol{g}$ outputs; the results are summarized in Table IV.

The angle of inclination of the accelerometer with respect to the foot is different for each subject, as the size and shape of shoe influence the orientation of the GaitShoe attachment on the shoe. When a subject stands still, with both feet flat on the floor (corresponding to a pitch of $0^{\circ}$ ), the angle of inclination of the $x$-accelerometer $\alpha_{x}$ can be determined as

$$
\alpha_{x}=\frac{\arcsin \vec{A}_{x}}{\vec{g}}
$$

where $\vec{A}_{x}$ is the acceleration measured by the $x$-accelerometer with the foot flat on the floor. The angle of inclination of the $y$-accelerometer $\alpha_{y}$ was similarly determined; the difference between these two angles provides the relative orientation between the $x$ - and $y$-accelerometers in the $x-y$ plane.

\section{Analysis}

1) Heel-Strike and Toe-Off (Initial): The primary use of the calibrated FSR data was to determine initial values of heel-strike and toe-off timing, which were then used to set the integration bounds used to calculate the pitch and stride length.
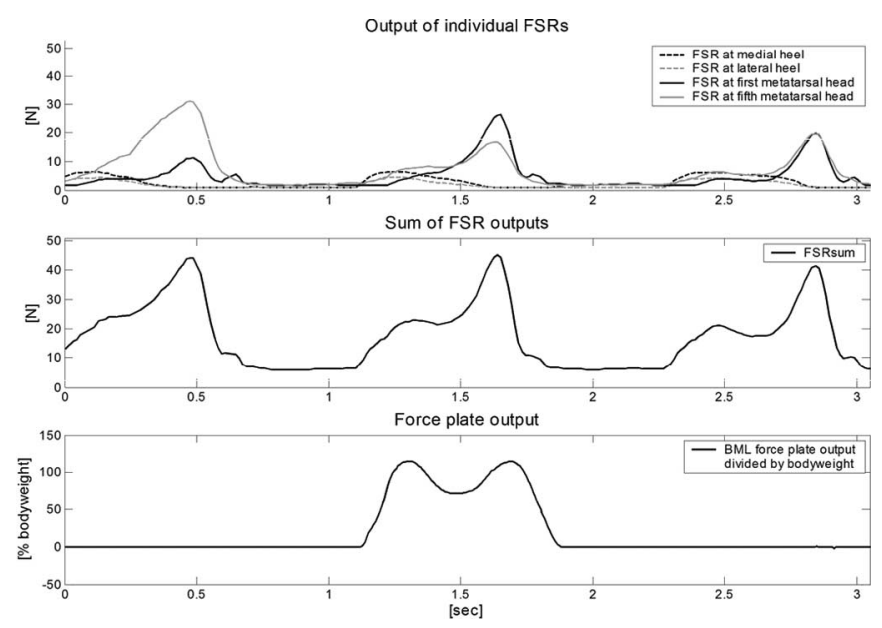

Fig. 7. Comparison of the calibrated FSR outputs to the force plate output.

A sample of the individual calibrated FSR outputs, the sum of the four calibrated FSR outputs, and the BML force plate output are shown in Fig. 7. The FSRs only cover a small percentage of the total weight-bearing area underneath the foot; as such, they only measure a portion of the total force. Thus, the shape of the summed FSR output in the middle graph is different than the bottom graph of the force plate measurement, because the summed FSR output is subsumed by the total force output.

A spline was fit to the FSRsum with time points every $1 \mathrm{~ms}$ to improve the time resolution. The time for heel-strike was set at the first time point (before the local maximum) with an FSRsum value that exceeded the previous by more than 0.005 $\mathrm{kg}$. The time for toe-off was set at the first time point (after the local minimum) with an FSRsum value within $0.005 \mathrm{~kg}$ of the following; these conditions were set after the inspection of many gait trials.

2) Pitch: The pitch of the foot $\Theta(t)$ was determined by integrating the $z$-gyroscope output. The sign of the pitch follows the convention used at the MGH BML, where a positive rotation about the $z$-axis corresponds to a rotation from the $y$-axis to the $x$-axis. The $z$-gyroscope output was integrated over single strides, with the integration bounds determined from the initial estimates of heel-strike and toe-off. The midpoints between the heel-strike and toe-off times were used as the bounds of integration; these midpoints occurred during the stance phase when the subject's foot was flat on the floor, so the initial value of the pitch was set to $0^{\circ}$.

To compensate for drift, an iterative method of integrating the $z$-gyroscope output was developed. The $z$-gyroscope data were integrated using trapezoidal integration, and the final value was compared to an "end-limit" value, defined as $0.1 \%$ of the full scale across all initial integrations. For a given step, if the final value had a magnitude greater than the end limit, a small "nudge" value was added to the calibrated $z$-gyroscope data, to adjust for changes in the zero offset, between these integration bounds. The magnitude of the nudge value was equal to the difference between the end limit and the final value after integration, and the sign of the nudge value was opposite from the sign of the final value after integration. This process was repeated until the 

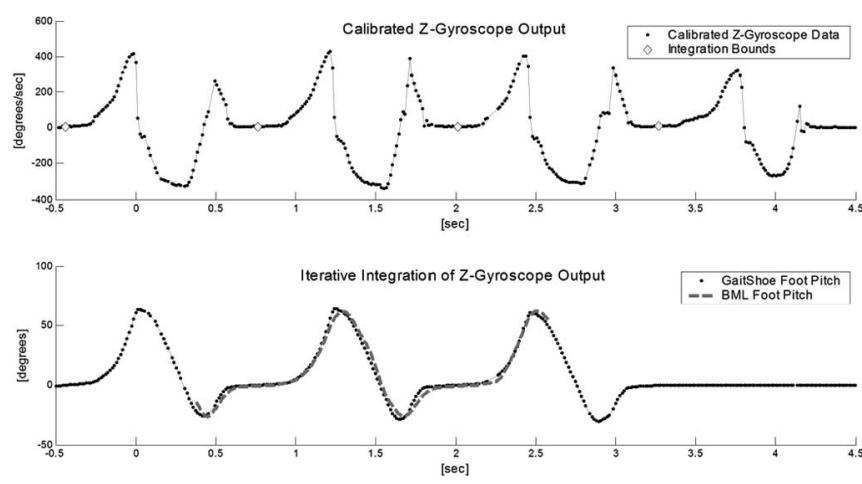

Fig. 8. Sample results of $z$-gyroscope integration.

final value was within the end limit. Results are shown in Fig. 8 for data collected from a left foot, with the corresponding data collected by the BML.

3) Velocity and Stride Length: Velocity and stride length were determined by single- and double-integration, respectively, of the kinematic acceleration along the $X_{\text {room }}$-axis, using the output of the $x$ - and $y$-accelerometers. For each accelerometer, the acceleration was resolved into two components, corresponding to the kinematic acceleration from foot motion, and the static acceleration due to gravity, i.e., $\vec{A}_{X-\mathrm{GS}_{\text {kinematic }}}$ and $\vec{A}_{X-\mathrm{GS}_{\text {static }}}$. To calculate the magnitude of the $\vec{A}_{X-\mathrm{GS}_{\text {static }}}$ component, the orientation of the $x$-accelerometer with respect to the room $\Phi_{x}$ was determined for every time point $t$

$$
\Phi_{x}\left(t_{i}\right)=\alpha_{x}+\Theta\left(t_{i}\right) .
$$

The linear acceleration measured by the $x$-accelerometer at time point $t_{i}, \vec{A}_{X-\mathrm{GS}_{\text {kinematic }}}\left(t_{i}\right)$ was calculated by subtracting the contribution of gravity from the total acceleration:

$$
\vec{A}_{X-\mathrm{GS}_{\text {kinematic }}}\left(t_{i}\right)=\vec{A}_{x}\left(t_{i}\right)-\vec{g} \sin \Phi_{x}\left(t_{i}\right) .
$$

The total acceleration vector experienced by the foot was resolved into two components in the reference frame of the room $\vec{A}_{X-\text { room }_{\text {kin }}}$ and $\vec{A}_{Y-\text { room }_{\text {kin }}}$, which contribute to the kinematic components measured by the accelerometers. The actual kinematic acceleration along $X_{\text {room }}$ was determined by combining the outputs of the $x$ - and $y$-accelerometers

$$
\begin{aligned}
\vec{A}_{X}-\text { room }_{\text {kinematic }} & \\
& =\frac{\vec{A}_{X-\mathrm{GS}_{\mathrm{dynamic}}} \sin \Phi_{y}-\vec{A}_{Y-\mathrm{GS}_{\mathrm{dynamic}}} \sin \Phi_{x}}{\cos \Phi_{x} \sin \Phi_{y}-\cos \Phi_{y} \sin \Phi_{x}} .
\end{aligned}
$$

$\vec{A}_{X-\text { room }_{\text {kinematic }}}$ was integrated twice: a single integration for the stride velocity and a subsequent integration for the stride length. The lower integration bound was determined by starting at the first quarter-point between a pair of $z$-gyroscope integration bounds, and stepping back in time toward the first $z$ gyroscope integration bound until the magnitude of $\vec{A}_{X-\mathrm{GS}_{\text {kinematic }}}$ was less than $0.2 \mathrm{~m} / \mathrm{s}^{2}$. If no value met this condition, the lower bound was set at the time point between the first $z$-gyroscope integration bound and the first quarter-point where the magnitude of $\vec{A}_{X-\mathrm{GS}_{\text {kinematic }}}$ was a local minimum. Similarly, the upper integration bound was determined by stepping forward in time

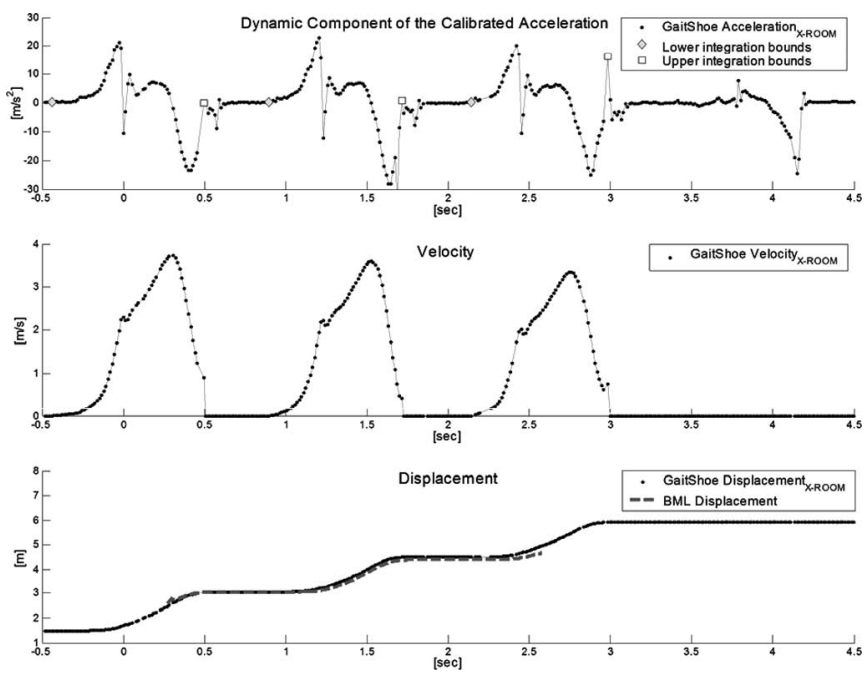

Fig. 9. Sample results of the accelerometer integration.

from the midpoint between two subsequent $z$-gyroscope integration bounds toward the second $z$-gyroscope integration bound, until $\vec{A}_{X-\mathrm{GS}_{\text {kinematic }}}$ had either a magnitude less than $0.2 \mathrm{~m} / \mathrm{s}^{2}$ or a value greater than $0 \mathrm{~m} / \mathrm{s}^{2}$. The latter condition was for the instances where a large positive acceleration was detected, corresponding to a strong heel-strike. If neither of these conditions were met, the upper bound was set at the time point between the second $z$-gyroscope integration bound and ten points prior where the magnitude of $\vec{A}_{X-G S_{\text {kinematic }}}$ was a local minimum.

The results of the integrations are shown in Fig. 9, with the corresponding displacement data collected by the BML, shifted to align with the GaitShoe results during the first available stance. The lower and upper integration bounds indicated on the $\vec{A}_{X-\text { room }_{\text {kinematic }}}$ plot.

\section{Heel-Strike and Toe-Off Timing (Final)}

Timing of heel-strike and toe-off were finalized using several sensors. The final value of the heel strike was changed if the upper integration bound for the $x$-accelerometer was set due to a magnitude of $\vec{A}_{X-\mathrm{GS}_{\text {kinematic }}}$ greater than $0 \mathrm{~m} / \mathrm{s}^{2}$. This second condition was expected to correspond to an especially strong heel-strike, and if present, that time point replaced the heelstrike time initially determined using FSRsum. The final values of the toe-off times were determined by the maximum pitch: normally, as the foot rolls off the floor, the pitch increases, and once the toe is off the ground, the start of leg-swing results in a decrease in the pitch.

\section{RESUlts}

The pitch, displacement, and heel-strike and toe-off timing as analyzed by the GaitShoe system were compared to data collected simultaneously by the MGH BML. The local maxima and minima of the pitch, the stride length, the heel-strike time, and the toe-off time for individual strides were compared directly (some trials had multiple strides available for comparison). In addition, the rms error between the pitch and displacement curves for both systems was calculated, using a spline fit to 
the GaitShoe pitch and displacement at time points corresponding to the BML data. Testing of the 15 subjects resulted in 270 total trials of gait. Finally, the GaitShoe results used in this validation study were separated into healthy gait and Parkinsonian gait groups.

The GaitShoe and BML data were analyzed for outlier data, and the BML data were checked for errors due to array slippage, and these data points were removed; in addition, the GaitShoe wireless protocol occasionally failed to receive a packet because of interference [36]. These causes resulted in small gaps in the data from both systems. Comparisons were excluded if there were more than ten individual gaps in the compared BML or GaitShoe data.

For pitch, each minimum and maximum was located, and a spline was fit over nine data points, with the measured extrema at the center. The spline was fit with time points every $3.34 \mathrm{~ms}$, and the new extrema and corresponding time point were determined. The stride lengths for each system were determined from the difference in displacement between two successive stance periods. The BML heel-strike and toe-off times were determined by one experienced physical therapist through computer-aided inspection of the BML force plate data; approximately, three heel-strike times and three toe-off times were determined for both feet of each subject, for a total of 86 comparisons. All direct comparisons are the value of the GaitShoe parameter minus the value of the BML parameter.

\section{A. RMS Comparison of Curves}

The mean rms error between the GaitShoe pitch and the BML pitch was $5.2 \pm 2.0^{\circ}$, calculated over 195 samples. The mean rms error between the GaitShoe displacement and the BML displacement, calculated over 303 samples, was $8.5 \pm 5.5 \mathrm{~cm}$.

\section{B. Direct Comparison of Peak Changes}

The mean difference between the GaitShoe pitch extrema and the BML pitch extrema was $-0.7 \pm 6.6^{\circ}$, and the mean percentage change was $15.6 \pm 18.4 \%$, calculated over 1132 samples. The mean difference between the time points of the extrema was $-26.0 \pm 24.2 \mathrm{~ms}$. The Pearson's correlation between the pitch extrema was 0.992, and the Pearson's correlation between the time points of the extrema was 1.000 .

The mean difference between the GaitShoe stride length and the BML stride length was $7.4 \pm 13.6 \mathrm{~cm}$, and the mean percent change was $6.5 \pm 11.7 \%$, calculated over 315 samples. The Pearson's correlation was 0.841 .

The mean difference between the GaitShoe heel-strike and the BML heel-strike times was $-6.7 \pm 22.9 \mathrm{~ms}$, over 77 samples. The mean difference between the GaitShoe toe-off and the BML toe-off times was $-2.9 \pm 16.9 \mathrm{~ms}$, over 75 samples. The Pearson's correlation was 0.999 between the heel-strike times and 1.000 between the toe-off times.

\section{Comparison of Healthy Gait and Parkinsonian Gait}

The means and standard deviation for each of the gait variables analyzed across healthy Parkinsonian gait groups are sum-
TABLE V

GAIT PARAMETERS BY SUBJECT GROUP

\begin{tabular}{lcccc}
\hline \hline & \multicolumn{2}{c}{ Healthy Gait } & \multicolumn{2}{c}{ Parkinsonian Gait } \\
Parameter & Mean $(\sigma)$ & Samples & Mean $(\sigma)$ & Samples \\
\hline Maximum Pitch $\left[{ }^{\circ}\right]$ & $70.1(6.6)$ & 282 & $56.6(10.4)$ & 290 \\
Minimum Pitch $\left[{ }^{\circ}\right]$ & $-29.3(5.3)$ & 331 & $-21.8(6.3)$ & 229 \\
\hline Stride Length $[\mathrm{m}]$ & $1.39(0.16)$ & 151 & $1.13(0.26)$ & 164 \\
\hline Stride Time $[\mathrm{s}]$ & $1.07(0.09)$ & 48 & $1.22(0.21)$ & 26 \\
Percent Stance Time $[\%]$ & $65.6(2.5)$ & 48 & $67.8(3.5)$ & 26 \\
\hline \hline
\end{tabular}

marized in Table V. For pitch extrema, the healthy persons' gait range is much larger than that of the Parkinsonian range, with a mean $14.5^{\circ}$ beyond for maximum pitch, and a mean $7.5^{\circ}$ beyond for minimum pitch. Correspondingly, the normal stride length is a mean $0.26 \mathrm{~m}$ longer than the Parkinsonian stride length. Though the stride time of the normal subjects is shorter by $0.15 \mathrm{~s}$, the percentage of the stride spent in stance is nearly equivalent, with the normal subjects spending only $2.2 \%$ less time in stance. The aforementioned differences in gait variables collected with the GaitShoe between healthy persons and persons with PD correlate with the data simultaneously collected by the BML.

\section{DISCUSSION}

The GaitShoe system costs under $\$ 500$ per foot in prototype quantities and the hardware for a single shoe weighs under $300 \mathrm{~g}$. The hardware is readily fixed to a variety of typical walking shoes, and data can be collected continuously over a few hours.

While the use of only four FSRs does not provide a full picture of the force distribution beneath the foot, the number is sufficient to provide a general picture of medial versus lateral force and heel versus metatarsal force. The comparison of heel force versus metatarsal force was used in determining stance time, as well as heel-strike and toe-off timing.

The coarse distribution of sensors does not render the FSR measurements useless: on the contrary, as shown in Fig. 7, though the shape of the FSRsum is similar across the three steps shown, there were substantial differences in the weight distribution between the first and fifth metatarsal heads. In the first step shown, more weight is on the fifth metatarsal, while in the second step, more weight is on the first metatarsal, and in the third step, the weight is distributed fairly evenly across the first and fifth metatarsal heads. This information cannot be obtained from a standard force plate.

The simplified kinematic analysis of the foot motion, using only the $x$ - and $y$-accelerometers and the $z$-gyroscope, resulted in reasonable estimations of the pitch and stride length.

The most significant contribution to the errors is likely to be from the $z$-gyroscope. The pitch, which is determined by integrating the $z$-gyroscope output, is used to both subtract the gravitational component of the acceleration and determine the kinematic component of the acceleration along the $X_{\text {room }}$ coordinate from the $x$ - and $y$-accelerometers. Thus, small errors in pitch have a compounded effect on the calculations of stride length, 
and reducing the standard deviation of the GaitShoe pitch measurement is likely to result in improved GaitShoe stride length calculations. Decreasing the time deviation in the pitch will also likely improve the stride length results.

An additional contribution to these errors is likely due to the simplified kinematic model, which assumed that all the motion of the foot was in a single plane, and that the $x$ - and $y$ accelerometers were positioned entirely within that single plane. Foot motion during gait is complex, and certainly exhibits accelerations and rotations outside of a single plane. In addition, it is unlikely that the $x$-and $y$-accelerometers were positioned entirely in a plane orthogonal to $X_{\text {room }}$ and $Y_{\text {room }}$. Thus, acceleration along the $Z_{\text {room-axis would have affected the } x \text { - and }}$ $y$-accelerometers, but not been accounted for, and the true orientation of the accelerometers with respect to the horizontal would be slightly different, resulting in a slightly incorrect calculation of the gravitational acceleration.

Though the inherent drift of the accelerometers and gyroscopes induce additional errors, gait analysis has the advantage that the integration is only carried out over a short time scale (under $0.5 \mathrm{~s}$, typically). The accuracy of the integration limits can be enhanced by improved force sensing underneath the foot to better determine when the foot transitions in and out of the phase where it is flat on the floor.

Even with only four FSRs, the multisensor GaitShoe determination of heel-strike and toe-off was highly successful, as compared to the BML heel-strike and toe-off time. The determination of the GaitShoe's toe-off time used the calculated pitch, so again, the improvement of the pitch will likely propagate through to improve the standard deviation of the toe-off time. The heel-strike time was determined either by the presence of a spike in the $x$-accelerometer, or, if no spike was present, by a threshold of the first difference of a spline fit to the FSRsum. Better calibration of the FSRsum, resulting in an objectively determined threshold, would likely improve this component of determining heel-strike; if so, placing FSRs underneath the great toe may be able to contribute to the determination of the toe-off time. Finally, increasing the data transfer rate of the GaitShoe from $75 \mathrm{~Hz}$ would eliminate the need to fit a spline to the FSRsum data, and may also improve the accelerometer and gyroscope data as well. The placement of additional IMU boards on the shins of the subject could provide useful information about the relative orientation between the foot and the shin, as well as about the orientation of the shin.

The validation results indicate that the GaitShoe can be further developed into a true wearable podiatric laboratory. It could allow the evaluation of persons who live in communities without access to a motion laboratory. The GaitShoe also offers the benefit of providing gait evaluation over longer periods of time versus the limitation of a one-time visit to a motion laboratory. Similarly, the GaitShoe can allow the evaluation to be carried out in a natural environment, such as in the home, offering clinicians a better understanding of a patient's daily gait pattern.

The primary purpose of this study was to evaluate the ability of the GaitShoe to perform basic gait analysis by validating its output with an established gait analysis system. As anticipated, the GaitShoe was able to recognize the differences between mean foot pitch extrema and gait stride time for healthy gait and those with PD, as also found in the BML data. Clinical interpretation of these gait differences is ongoing.

\section{CONCLUSION AND FUTURE RESEARCH}

These initial results demonstrate that the GaitShoe promises to be an important research tool, capable of enabling the analysis of gait in untraditional ways, such as over long time periods and in the home environment or through the use of multisensor pattern recognition. Fast processing of the data stream can provide real-time feedback for use in applications such as sports medicine, electrostimulation, or physical therapy.

Additional research with the GaitShoe sensor outputs has involved the application of standard pattern recognition techniques to discriminate between healthy gait and Parkinsonian gait, as well as to discriminate between individuals [36] and real-time analysis of the data to provide therapeutic musical feedback to investigate interactive applications in physical therapy [36], [45]. Future research to improve the GaitShoe will focus on extending the IMU analysis to utilize the full $6 \mathrm{DOF}$, on improving the wireless transmission, and changing the conditioning electronics for the FSRs [46]. For the IMU analysis, calibration routines to determine the actual orientation of each of the axes of the accelerometers and gyroscopes will be implemented, and an optimal estimator such as a Kalman filter will be used to analyze the data [47]. Recent devices, such as the ADXL330 triaxial accelerometer, and dual-axis gyros such as the Intelli-G chips from Invensense promise to simplify and miniaturize the GaitShoe's IMU significantly in future designs. New strategies for wireless transmission will be investigated, encompassing both newly available devices, as well as alternate locations for the antennae with reduced interference from the human body [48]. For experiments that do not require real-time data analysis, onboard data storage will be considered [40]. We are also developing dynamic sensor-driven power management techniques to allow these multimodal wearable systems to significantly extend their battery life [49].

The research involving therapeutic musical feedback is ongoing, particularly for patients with Parkinsonian gait. Other extensions to this paper include placing IMUs on additional body locations, such as the shin and thigh, as well as the upper body, to generate more information about the motion of the subject. In addition, the equipment developed for the GaitShoe has a potential benefit for areas of research, such as ergonomics, that would benefit from real-time analysis of motion, but do not have ready access to traditional motion analysis equipment.

\section{ACKNOWLEDGMENT}

The authors thank their colleagues at the MGH Biomotion Laboratory and the MIT Media Laboratory, and J. Memishian at Analog Devices for generously providing samples of the prototype version of the ADXRS150 gyroscope. They appreciate the enthusiasm and support from the MGH Neurology Department, especially L. Shinobu, M.D., Ph.D., and S. Parker, M.D. They are grateful to the volunteers for the subject testing and thank them for their generous contribution. 


\section{REFERENCES}

[1] H. S. Zhu, N. Maalej, J. G. Webster, W. J. Tompkins, P. Bach-y-Rita, and J. J. Wertsch, "An umbilical data-acquisition system for measuring pressures between the foot and shoe," IEEE Trans. Biomed. Eng., vol. 37, no. 9, pp. 908-911, Sep. 1990.

[2] H. S. Zhu, J. J. Wertsch, G. F. Harris, J. D. Loftsgaarden, and M. B. Price, "Foot pressure distribution during walking and shuffling," Arch. Phys. Med. Rehabil., vol. 72, no. 6, pp. 390-397, May 1991.

[3] H. S. Zhu, J. J. Wertsch, G. F. Harris, H. M. Alba, and M. B. Price, "Sensate and insensate in-shoe plantar pressures," Arch. Phys. Med. Rehabil., vol. 74, no. 12, pp. 1362-1368, Dec. 1993.

[4] J. M. Hausdorff, Z. Ladin, and J. Y. Wei, "Footswitch system for measurement of the temporal parameters of gait," J. Biomech., vol. 28, no. 3, pp. 347-351, Mar. 1995.

[5] J. M. Hausdorff, L. Zemany, C.-K. Peng, and A. L. Goldberger, "Maturation of gait dynamics: Stride-to-stride variability and its temporal organization in children," J. Appl. Physiol., vol. 86, no. 3, pp. 1040-1047, Mar. 1999.

[6] J. M. Hausdorff, D. A. Rios, and H. K. Edelberg, "Gait variability and fall risk in community-living older adults: A 1-year prospective study," Arch. Phys. Med. Rehabil., vol. 82, no. 8, pp. 1050-1056, Aug. 2001.

[7] J. M. Hausdorff, P. L. Purdon, C.-K. Peng, Z. Ladin, J.-Y. Wei, and A. L. Goldberger, "Fractal dynamics of human gait: Stability of long-range correlations in stride interval fluctuations," J. Appl. Physiol., vol. 80, pp. 1448-1457, 1996.

[8] R. E. Morley, E. J. Richter, J. W. Klaesner, K. S. Maluf, and M. J. Mueller, "In-shoe multisensory data acquisition system," IEEE Trans. Biomed. Eng., vol. 48, no. 7, pp. 815-820, Jul. 2001.

[9] K. S. Maluf, R. E. Morley, E. J. Richter, J. W. Klaesner, and M. J. Mueller, "Monitoring in-shoe plantar pressures, temperature, and humidity: Reliability and validity of measures from a portable device," Arch. Phys. Med. Rehabil., vol. 82, no. 8, pp. 1119-1127, Aug. 2001.

[10] I. P. Pappas, M. R. Popovic, T. Keller, V. Dietz, and M. Morari, "A reliable gait phase detection system," IEEE Trans. Neural Syst. Rehabil. Eng., vol. 9, no. 2, pp. 113-125, Jun. 2001.

[11] I. P. Pappas, T. Keller, and S. Mangold, "A reliable, gyroscope based gait phase detection sensor embedded in a shoe insole," presented at the 2002 IEEE Int. Conf. Sens., Orlando, FL.

[12] E. Vildjiounaite, E.-J. Malm, J. Kaartinen, and P. Alahuhta, "Location estimation indoors by means of small computing power devices, accelerometers, magnetic sensors, and map knowledge," Lect. Notes Comput. Sci., vol. 2414, pp. 211-224, Jan. 2002.

[13] R. G. Cutlip, C. Mancinelli, F. Huber, and J. DiPasquale, "Evaluation of an instrumented walkway for measurement of the kinematic parameters of gait," Gait Posture, vol. 12, no. 2, pp. 134-138, Oct. 2000.

[14] C. Giacomozzi and V. Macellari, "Piezo-dynamometric platform for a more complete analysis of foot-to-floor interaction," IEEE Trans. Rehabil. Eng., vol. 5, no. 4, pp. 322-330, Dec. 1997.

[15] C. D. Kidd, R. Orr, G. D. Abowd, C. G. Atkeson, I. A. Essa, B. MacIntyre, E. Mynatt, T. E. Starner, and W. Newstetter, "The aware home: A living laboratory for ubiquitous computing research," in Proc. CoBuild 1999: 2nd Int. Conf. Cooperative Buildings, pp. 191-198.

[16] J. Suutala, S. Pirttikangas, J. Riekki, and J. Röning, "Reject-optional LVQbased two-level classifier to improve reliability in footstep identification," in Proc. 2004 Conf. Pervasive Comput., pp. 182-187.

[17] J. Davis and H. Gao, "An expressive three-mode principal components model of human action style," Image Vis. Comput., vol. 21, no. 11, pp. 1001-1016, 2003.

[18] S. A. Niyogi and E. H. Adelson, "Analyzing gait with spatiotemporal surfaces," in Proc. IEEE Workshop Nonrigid Articulated Motion, Austin, TX, Nov. 1994, pp. 64-69.

[19] C. Y. Yam, M. S. Nixon, and J. N. Carter, "Automated person recognition by walking and running via model-based approaches," Pattern Recog., vol. 37, no. 5, pp. 1057-1072, 2004.

[20] A. Y. Johnson, J. Sun, and A. F. Bobick, "Predicting large population data cumulative match characteristic performance from small population data," in Proc. 4th Int. Conf. Audio-Video-Based Biometric Person Authentication, Guildford, U.K., Jun. 2003, pp. 1061-1062.

[21] D. Voth, "You can tell me by the way I walk-In the news," IEEE Intell. Syst., vol. 18, no. 1, pp. 4-5, Jan./Feb. 2003.

[22] Walk the walk: Gait recognition technology could identify humans at a distance. Georgia Tech. Res. News [Online]. Available: http://gtresearchnews.gatech.edu/newsrelease/GAIT.htm.
[23] J. A. Paradiso, "Electronic music: New ways to play," IEEE Spectrum, vol. 34, no. 12, pp. 18-30, Dec. 1997.

[24] [Online]. Available: http://www.pro-balance.com.

[25] [Online]. Available: http://www.zeek.com/games/redlight/.

[26] [Online]. Available: http://www.fitsense.com/.

[27] [Online]. Available: http://www.vectrasense.com/.

[28] [Online]. Available: http://www.adidas.com/.

[29] [Online]. Available: http://www.nike.com/.

[30] I. Choi and C. Ricci, "Foot-mounted gesture detection and its application in virtual environments," in Proc. IEEE Int. Conf. Syst., Man, Cybern., Comput. Cybern. Simul., Oct. 1997, vol. 5, pp. 4248-4253.

[31] Tekscan, Inc. (2004). F-scan system features [Online]. Available: http://www.tekscan.com/medical/specs_fscan $1 . h t m l$.

[32] [Online]. Available: http://www.clevemed.com/.

[33] [Online]. Available: http://www.portablegaitlab.com/.

[34] J. A. Paradiso, K. Hsiao, A. Y. Benbasat, and Z. Teegarden, "Design and implementation of expressive footware," IBM Syst. J., vol. 39, no. 3, pp. 511-519, 2000.

[35] J. D'Agnese, "The 11th annual discover awards," Discover, vol. 21, no. 7, pp. 89-112, 2000.

[36] S. J. Morris, "A shoe-integrated sensor system for wireless gait analysis and real-time therapeutic feedback," Sc.D. dissertation, Harvard/MIT Division Health Sci. Technol., Cambridge, MA, Jun. 2004.

[37] A. Lawrence, Modern Intertial Technology. New York: Springer-Verlag, 1993.

[38] Motorola, Inc. (2003, February). MC33794 data sheet, Rev 6.0 [Online]. Available: http://wwww.motorola.com./files/analog/doc/ data_sheetMC33794.pdf.

[39] A. Y. Benbasat, S. J. Morris, and J. A. Paradiso, "A wireless modular sensor architecture and its application in on-shoe gait analysis," in Proc. IEEE Int. Conf. Sensors, Oct. 21-24, 2003, pp. 1086-1091.

[40] A. Y. Benbasat and J. A. Paradiso, "A compact modular wireless sensor platform," in Proc. Symp. Inf. Proc. Sensor Netw. (ISPN), Los Angeles, CA, Apr. 25-27, 2005, pp. 410-415.

[41] E. K. Antonnson, "A three-dimensional kinematic acquisition and intersegmental dynamic analysis system for human motion," Ph.D. dissertion, Dept. Mech. Eng., MIT, Cambridge, MA, 1982.

[42] P. O. Riley, R. W. Mann, and W. A. Hodge, "Modelling of the biomechanics of posture and balance," J. Biomech., vol. 23, no. 5, pp. 503-506, 1990.

[43] P. O. Riley, M. L. Schenkman, R. W. Mann, and W. A. Hodge, "Mechanics of a constrained chair-rise," J. Biomech., vol. 24, no. 1, pp. 77-85, 1991.

[44] M. E. Morris, F. Huxham, J. McGinley, K. Dodd, and R. Iansek, "The biomechanics and motor control of gait in Parkinson disease," Clin. Biomech., vol. 16, no. 6, pp. 459-470, Jul. 2001.

[45] J. A. Paradiso, S. J. Morris, A. Y. Benbasat, and E. Asmussen, "Interactive therapy with instrumented footwear," in Proc. Comput. Human Interact. (CHI) 2004, Vienna, Austria, Apr. 24-29, pp. 1341-1343.

[46] S. J. M. Bamberg, P. LaStayo, L. Dibble, J. Musselman, and S. Kiran, "Development of a quantitative in-shoe measurement system for assessing balance: Sixteen-sensor insoles," in Proc. IEEE 2006 Int. Conf. Eng. Med. Biol. Soc., Aug. 30-Sept. 3, pp. 6041-6044.

[47] N. El Sheimy, E.-W. Shin, and X. Niu, "Kalman filter face-off: Extended vs. unscented Kalman filters for integrated GPS and MEMS inertial," InsideGNSS, vol. 1, no. 2, pp. 48-54, Mar. 2006.

[48] R. Aylward and J. A. Paradiso, "A compact, high-speed, wearable sensor network for biomotion capture and interactive media," in Proc. 6th Int. Conf. Inf. Proc. Sensor Netw. (IPSN 2007), Cambridge, MA, Apr. 25-27, pp. 380-389.

[49] A. Y. Benbasat and J. A. Paradiso, "Groggy wakeup—Automated generation of power-efficient detection hierarchies for embedded sensors," in Proc. Int. Workshop Wearable Implantable Body Sensor Netw. (BSN 2007), Aachen, Germany, Mar. 26-28, pp. 59-64.

Stacy J. Morris Bamberg (S'02-M'05) received the S.B. and S.M. degrees in mechanical engineering from the Massachusetts Institute of Technology (MIT), Cambridge, in 1996 and 1999, respectively, and the Sc.D. degree in medical engineering from the Harvard/MIT Division of Health Sciences and Technology, Cambridge, in 2004.

She is currently an Assistant Professor of Mechanical Engineering at the University of Utah, Salt Lake City. 
Ari Y. Benbasat ( $\left.\mathrm{S}^{\prime} 00-\mathrm{M}^{\prime} 07\right)$ received the B.A.Sc. degree in engineering physics from the University of British Columbia, Vancouver, BC, Canada, in 1998, and the Ph.D. degree in media arts and sciences from the Massachusetts Institute of Technology (MIT), Cambridge, in 2007.

$\mathrm{He}$ is currently with the Massachusetts Institute of Technology (MIT) Media Laboratory, Cambridge, where he was engaged in research on design methodologies for the creation of real-time power optimal wearable sensors.

Donna Moxley Scarborough received the B.Sc. degree in physical therapy from the Northeastern University, Boston, MA, in 1988, and the M.Sc. degree from the Massachusetts General Hospital (MGH) Institute of Health Professions, Boston, in 1997.

He was an Assistant Clinical Director of the MGH Biomotion Laboratory, and is currently a Clinical Research Program Manager and Research Physical Therapist at the MGH Orthopedics Biomechanics and Biomaterials Laboratory, Boston.
David E. Krebs received the B.S. degree in physical therapy and the M.A. degree in applied physiology from Columbia University, New York, in 1977 and 1979 respectively, the Ph.D. degree in pathokinesiology and physical therapy from New York University, New York, in 1986, and the D.P.T. degree in physical therapy from the Massachusetts General Hospital (MGH) Institute of Health Professions, Boston, in 2002.

He was engaged in orthopedic surgery at the Harvard Medical School, Boston, MA, and in mechanical engineering at the Massachusetts Institute of Technology (MIT), Cambridge.

Joseph A. Paradiso (M'89-SM'07) received the B.S. degree (summa cum laude) in electrical engineering and physics from Tufts University, Medford MA, in 1977, and the Ph.D. degree in physics from the Massachusetts Institute of Technology (MIT), Cambridge, in 1981.

In 1994, he joined the MIT Media Laboratory, where he is currently a Sony Career Development Associate Professor of media arts and sciences and directs the Responsive Environments Group. 